\title{
Understanding morphology-mobility dependence in PEDOT:Tos
}

\author{
Nicolas Rolland, ${ }^{1}$ Juan Felipe Franco-Gonzalez, ${ }^{1}$ Riccardo Volpi, ${ }^{2,3}$ Mathieu Linares, ${ }^{4,5}$ and Igor V. Zozoulenko ${ }^{1, *}$ \\ ${ }^{1}$ Laboratory of Organic Electronics, Department of Science and Technology (ITN), Campus Norrköping, Linköping University, \\ SE-60174 Norrköping, Sweden \\ ${ }^{2}$ Department of Physics, Chemistry and Biology (IFM), Linköping University, SE-58183 Linköping, Sweden \\ ${ }^{3}$ Machine Learning and Optimization Group, Romanian Institute of Science and Technology (RIST), Cluj-Napoca, Romania \\ ${ }^{4}$ School of Biotechnology, Division of Theoretical Chemistry \& Biology, KTH Royal Institute of Technology, 11421 Stockholm, Sweden \\ ${ }^{5}$ Swedish e-Science Research Centre, KTH Royal Institute of Technology, 10450 Stockholm, Sweden
}

(Received 23 January 2018; revised manuscript received 15 March 2018; published 30 April 2018)

\begin{abstract}
The potential of conjugated polymers to compete with inorganic materials in the field of semiconductor is conditional on fine-tuning of the charge carriers mobility. The latter is closely related to the material morphology, and various studies have shown that the bottleneck for charge transport is the connectivity between well-ordered crystallites, with a high degree of $\pi-\pi$ stacking, dispersed into a disordered matrix. However, at this time there is a lack of theoretical descriptions accounting for this link between morphology and mobility, hindering the development of systematic material designs. Here we propose a computational model to predict charge carriers mobility in conducting polymer PEDOT depending on the physicochemical properties of the system. We start by calculating the morphology using molecular dynamics simulations. Based on the calculated morphology we perform quantum mechanical calculation of the transfer integrals between states in polymer chains and calculate corresponding hopping rates using the Miller-Abrahams formalism. We then construct a transport resistive network, calculate the mobility using a mean-field approach, and analyze the calculated mobility in terms of transfer integrals distributions and percolation thresholds. Our results provide theoretical support for the recent study [Noriega et al., Nat. Mater. 12, 1038 (2013)] explaining why the mobility in polymers rapidly increases as the chain length is increased and then saturates for sufficiently long chains. Our study also provides the answer to the long-standing question whether the enhancement of the crystallinity is the key to designing high-mobility polymers. We demonstrate, that it is the effective $\pi-\pi$ stacking, not the long-range order that is essential for the material design for the enhanced electrical performance. This generic model can compare the mobility of a polymer thin film with different solvent contents, solvent additives, dopant species or polymer characteristics, providing a general framework to design new high mobility conjugated polymer materials.
\end{abstract}

DOI: 10.1103/PhysRevMaterials.2.045605

\section{INTRODUCTION}

Organic conducting polymers are appealing materials for the semiconductors industry, which is currently essentially based on inorganic semiconductors such as silicon. They are not only progressively reaching similar material performance, but they also possess many properties that conventional semiconductors are missing, such as being easily processed, cheap, abundant, and environment friendly and having the mechanical properties of plastic, opening the door to the wide field of flexible and printed electronics [1]. They already proved to be efficient in a wide range of applications such as optoelectronics [2], organic field-effect transistors [3], electrochemical transistors [4], photovoltaics [5], sensors [6], and thermoelectricity [7], just to name a few. One of the most studied conducting polymer is poly-(3,4-ethylenedioxythiophene) (best known as PEDOT), which importance for organic electronics stems for its stability, well-developed manufacturing technology, and excellent electronic and optical properties [8-12]. It also supports ionic transport and is biocompatible, which makes

\footnotetext{
*igor.zozoulenko@liu.se
}

it possible to use it for bioelectronic application such as ion pumps [13] and drug delivery systems [14], as well as for energy storage applications such as supercapacitors, batteries, and fuel cells [15].

Despite considerable experimental progress accumulated since the mid-2000s, the development of conducting polymers has been hindered by a lack of fundamental knowledge about the physical processes behind conductivity in these materials, which makes difficult a rational and systematic material design. In particular, the central question on how the electron mobility in PEDOT can be understood and theoretically predicted from the polymer structure and morphology still remains unanswered and highly controversial. Recent studies show the premise of a unified model to describe these materials. For example, in a recent review it was stated that the chain length $N$ has a tremendous impact on the mobility [16]. This, however, is not fully consistent with a recent study of Noriega et al. [17], where, based on an extensive literature survey, it was demonstrated that the mobility of various conducting polymers is rather insensitive to the molecular weight (i.e., the chain length) but exhibits a sharp decrease for low weights for some polymers. This behavior was correlated with the degree of paracrystallinity (defined as the standard deviation of local 
static lattice fluctuations normalized by the average value of the lattice spacing) in the films and has been explained by invocating the existence of crystalline domains linked by tie molecules that cross an amorphous polymer matrix. A related important question which is currently under debate is whether one needs to improve crystallinity of the polymer in order to enhance its mobility [17-19].

It is remarkable that despite this great interest to unravel the relation between morphology and electrical performance, there is no reliable model that could guide the design of highperforming PEDOT materials based on its actual morphology. Indeed, so far theoretical description concerned with PEDOT and related systems utilized models not relying on its structural properties, which include cubic-grid Monte Carlo models [20,21], semi-analytical approaches [20-23] or idealized models of perfect crystal structure [24-26]. It is also noteworthy that Kang and Snyder [27] reported recently a general description of transport in conducting polymers that can capture essential transport parameters through a phenomenological single transport function [27]. Interestingly, the latter is a power law with exponent $s=3$ for most polymers except for PEDOT doped with Tosylate (PEDOT:Tos), where $s=1$. This makes PEDOT:Tos an interesting and important case study to unravel fundamental transport mechanisms in conducting polymers.

In contrast to the case on the above-mentioned theoretical approaches used for PEDOT, transport modeling from realistic morphologies obtained by molecular dynamics has been successfully applied to a wide range of organics materials with various levels of theory [28-40]. The cornerstone of these approaches is the calculation of the transfer integrals distribution in the materials to relate the film morphology to the carrier mobility, highlighting the bottlenecks for charge transport. For instance, in discotic liquid crystals the transport is limited by the tail of small transfer integrals (defects) [41], while in amorphous polypyrrole the transport is limited by a threshold transfer integral corresponding to the first peak in the distribution, rather insensitive to the global morphology [31].

Herein, we present a multiscale computational model to predict the charge carriers mobility in polymer films depending on their morphologies. The level of theory is kept as simple as possible so that the link between morphology and mobility is straightforward, and a general framework is provided to discuss transport in conducting polymers in terms of transfer integrals distribution and percolation. The model is applied to PEDOT:Tos, with particular emphasis on the effects of molecular weight and solvent content. Our results provide theoretical support of the findings of Noriega et al. [17] explaining why the mobility rapidly increases as the chain length is increased and then saturates for sufficiently long chains. Our study also provides the answer to the long-debated question regarding whether the enhancement of the crystallinity is the key to designing high-mobility polymers. We demonstrate that it is the effective $\pi-\pi$ stacking, not the long-range order, that is essential for the material design for enhanced electrical performance.

The organization of the article is as follows. In Sec. II we introduce our model of a resistive network based on the polymer morphology and we explain how the parameters of the model are calculated. In Sec. III, we develop the methodology to solve the model's equations and we present the results of the mobility dependence as a function of the chain length and water content. Finally, the last section is a discussion of the results that highlights the link between polymer morphology, transfer integral distribution, percolation, and mobility.

\section{RESULTS AND DISCUSSION}

\section{A. From morphology to resistive network}

The different PEDOT:Tos morphologies studied in this work were generated by molecular dynamics simulations (MD) following the methodology developed in previous works [42-44]. The initial systems are mixtures of positively charged PEDOT oligomers, Tosylate anions and water molecules; the system has a neutral charge when the total positive charge carried by the PEDOT oligomers exactly compensate the Tosylate anionic charge. The initial water concentration is set to $W=82 \% \mathrm{w} / \mathrm{w}$, and the crystallization of PEDOT oligomers and evolution of the morphology of the system is investigated by consequently removing a part of the water molecules and iteratively equilibrating the system at ambient temperature from the initial highly hydrated phase to a dry phase until no water remains in the same way at this has been done in Ref. [42] (see the Supporting Information for details [45]).

In this work, we focused on the systems with $W=$ $43 \%, 13 \%$, and $0 \% \mathrm{w} / \mathrm{w}$ (typical water concentration is 10 $20 \%$ in thin films under ambient conditions). The ratio of monomers in PEDOT chains to Tosylate anions is 3 to 1 , which corresponds to a pristine (i.e., as polymerized) PEDOT with the oxidation level $\approx 33 \%$ [46]. In each system, the chain length $N$ (number of monomers per chain) is constant, and the number of PEDOT chains is such that the total monomer number is close to 600 . We investigated the chain length impact on mobility by considering systems with $N=3,6,9,12,15$, and 18 . (Note that the experimental value for PEDOT chain length is not known exactly and estimated to be $N=5-15$ monomer units) $[47,48]$. A representative morphology obtained through this procedure is presented in Fig. 1(a).

Once the system is equilibrated by MD simulations, it can be converted into a resistive network with the following procedure. To define the network we first have to specify the origin of charge carriers in PEDOT. As specified above, we consider a heavily doped PEDOT with oxidation level $33 \%$, where three monomer units accommodate a positive charge $+e$. (Note that negative Tos counterions compensating positive charges in the polymer backbone, do not participate in the electrical transport). The positive charges correspond to the polaronic/bipolaronic band of PEDOT's electronic structure as schematically shown in Fig. 1(e) (for details of the electronic structure of PEDOT:Tos see Refs. [50,51]). A PEDOT doped with negative counterions represents a $p$-type material where electrical transport is due to the thermally assisted hopping of polarons/bipolarons via the states in the valence band. The tail of the density of states (DOS) in the valence band can be approximated by the Gaussian with a typical broadening $\sigma \sim 0.1 \mathrm{eV}$ as shown in Fig. 1(e) [23,50,51].

Based on the picture outlined above, a resistive network is built to model the diffusion of the charge carriers through the system. Each node of the network (also called a transport unit), belongs to a particular chain and represents an electronic state 
(a)

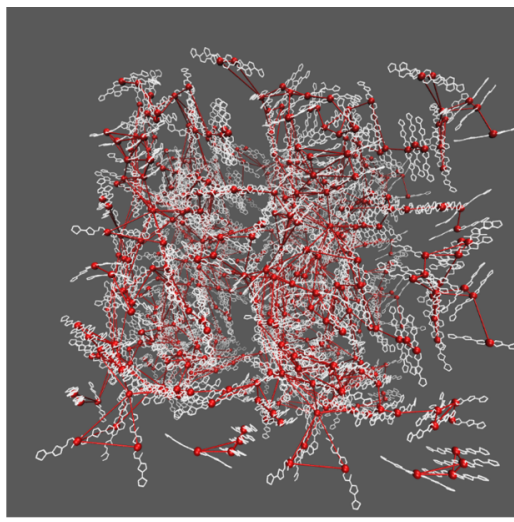

(c) Node 1: $\overrightarrow{\mathrm{r}_{1}}, \mathrm{E}_{1} \quad$ Node 2: $\overrightarrow{\mathrm{r}_{2}}, \mathrm{E}_{2} \quad \square($ (e)
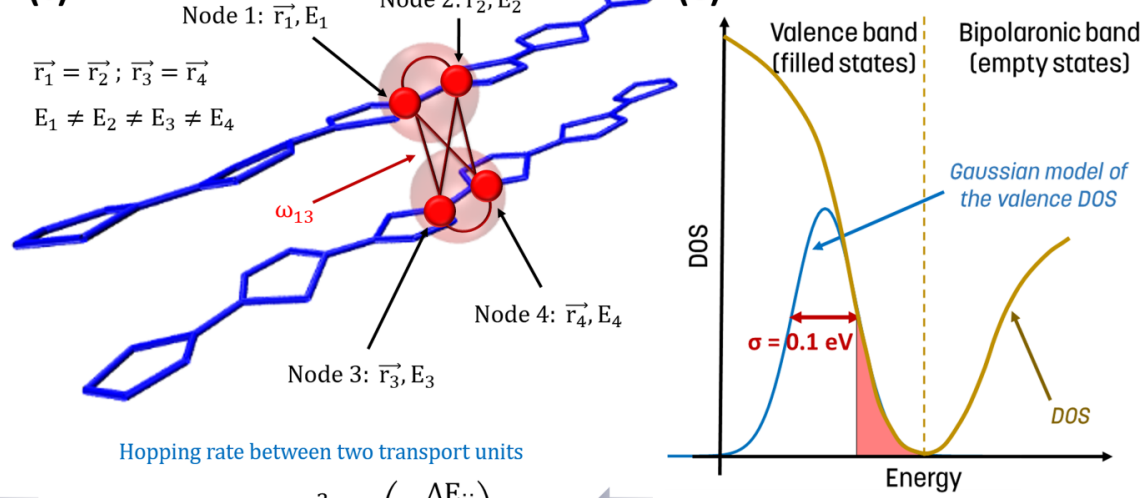

Hopping rate between two transport units

$$
\omega_{\mathrm{ij}}=\omega_{0}\left|\mathrm{H}_{\mathrm{ij}}\right|^{2} \exp \left(-\frac{\Delta \mathrm{E}_{\mathrm{ij}}}{\mathrm{k}_{\mathrm{B}} \mathrm{T}}\right)
$$

Energy difference

Truncated gaussian distribution

(b)

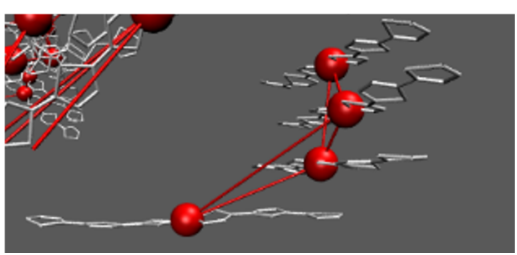

(d)

Transfer Integral Projection method, ZINDO level

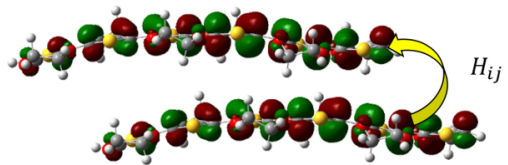

$$
\Delta E_{i j}=E_{j}-E_{i}-e \vec{F} \cdot \overrightarrow{R_{i j}}
$$

$E_{j}, E_{i}$ : Site energy

$\overrightarrow{\mathrm{F}}$ : Electric field

$\overrightarrow{\mathrm{R}_{\mathrm{ij}}}$ : Vector from node $i$ to $j$

FIG. 1. Workflow to transform molecular dynamics simulations into resistive networks. (a) Morphology obtained from MD simulation $(N=6$ and $W=43)$ and corresponding resistive network. Only thiophene rings (white) are shown for clarity. The red lines are the edges connecting the network nodes (PEDOT geometrical centers). (b) Zoom on a small part of the structure. (c) N/3 nodes are superimposed on each PEDOT chain. For each edge between two nodes a hopping rate is calculated according to a modified Miller-Abrahams expression. (d) Transfer integrals needed to calculate the hopping rates are calculated by the projection method at the ZINDO level. (e) Molecular orbital (MO) energies are drawn from a truncated gaussian distribution that represents the tail of the valence band DOS. The energy difference between MOs takes into account the electric field intensity. Molecular images were prepared using Visual Molecular Dynamics (VMD) [49].

of the valence band that can accommodate a charge carrier. In principle, each state of the valence band may therefore be included as one node in the network. However, to reduce the complexity of the network only a subset of these states corresponding to the tail of the valence band can be taken into account [red shaded area of the valence band in Fig. 1(e)], because the deep levels are not expected to contribute to the transport. In our model all systems considered with a chain length $N=3,6,9,12,15,18$ are treated on equal footing and thus they have the same number of nodes (this condition is justified because all systems considered have the same number of monomers and thus should be able to accommodate the same number of charge carriers). As a result, a number of nodes on a chain depends on its length, which is also consistent considering that we want to take into account the same energy window of the valence band for the different systems (note that the level spacing in the DOS is decreasing with the increase of the chain length). For each chain, we choose the number of nodes equals to $N_{\text {nodes }}=N / 3$. This is the minimum number of nodes to ensure that in the system with the shortest chains $(N=3)$ the transport can occur via hopping of polarons from one chain to the other (in this case one chain can accommodate $N / 3=1$ charge carrier). On the other hand, it seems reasonable to limit the number of charge carriers to 1 per 3 monomers, which corresponds to a typical oxidation level in a pristine (i.e., as polymerized) PEDOT.

Ideally, the geometrical positions of the $N_{\text {nodes }}$ nodes on one chain should be related to the carriers localization on the chain.
Indeed, for long chains or for noncoplanar polymers the polaron/bipolaron states can be strongly localized at the finite part of a chain [31]. However in PEDOT where chains are relatively short, it has been shown that the carriers can be delocalized over one entire single chain [50]. Thus, for simplicity, we choose a spatial position of all nodes in the geometrical centers of corresponding PEDOT chains. In addition, in our model the intermolecular transport is considered to be the limiting step for transport as the transport along one single chain backbone is really fast; therefore, there is no need to introduce spatially separated node on the same chain to model the transport. Now, edges connecting the nodes are introduced in the network to allow one carrier to hop from one node to the other. Two nodes are connected by an edge if for the corresponding pair of chains, two of their monomers are within a distance below a given threshold $d_{t}$, where we set $d_{t}=8,8.4,10.2 \mathrm{~nm}$, respectively, for $W=0,13,43$ [see Figs. 1(a) and 1(b)]. The threshold $d_{t}=8 \mathrm{~nm}$ for $W=0$ is slightly above a double $\pi-\pi$ stacking distance, and for higher distances the transfer integral is negligible and does not contribute to the transport (this is clear from the plot presented in the Results section); however, to treat all systems on the equal footing, the threshold was adjusted to take into account the decreasing molecule density in the higher solvent contents. If two nodes belong to the same chain, they are immediately connected by an edge. Considering two chains of $N=6$ as an example, there are $N / 3 * N / 3=4$ edges connecting the nodes belonging to different chains, and $N / 3 *[N / 3-1)]=2$ edges connecting the nodes on the same chain [see Fig. 1(c), for the sake of clarity the nodes on the 
same chain have been spatially separated but their coordinates are the same]. Hopping rates $\omega_{i, j}$ for the charge carriers are associated to each edge within a modified Miller-Abrahams formalism [52]:

$$
\omega_{i, j}=\omega_{0}\left|H_{i, j}\right|^{2} \exp \left(\frac{-\Delta E_{i, j}}{k_{B} T}\right)
$$

where $\omega_{0}$ is a prefactor, $H_{i, j}$ is the so-called transfer integral between states corresponding to the nodes $i$ and $j, \Delta E_{i, j}$ is the energy difference between states $i$ and $j, \Delta E_{i, j}=$ $E_{j}-E_{i}-e F r_{i, j}$, with $F$ being the electric field intensity and $r_{i, j}$ the distance in the field direction between the center of the chains holding nodes $i$ and $j$. The $E_{i}$ are the energies of the valence band states represented by the nodes: They are drawn from a truncated Gaussian distribution of standard deviation $0.1 \mathrm{eV}$ as depicted in Fig. 1(e) (only energies above $0.1 \mathrm{eV}$ from the Gaussian mean value are taken into account). Here it may be pointed out that the energy levels associated with neighboring nodes might be correlated because the electrostatic potential around the PEDOT chain is correlated with the positions of the counterions [50,51]. To keep our model on a tractable level we do not account for such correlation because this would require full quantum mechanical calculation over the whole system. For calculation of the transfer integral $H_{i, j}$ between nodes belonging to different chains, we made the approximation that it can be treated as the transfer integral between the molecular orbitals (MOs) of the neutral states of the chains. We use the dimer projection method at the ZINDO level [53], such that the relative orientation of two molecules is accounted for in the transfer integral values (as in Ref. [41]), see Fig. 1(d) for illustration. (Note that all corresponding MOs are calculated using GAUSSIAN09 [54] package for PEDOT chains as obtained from the MD simulations; the transfer integrals are evaluated for the neutral chains for $N / 3$ highest MOs in the valence band.) It should be stressed that ZINDO calculations give the expression for the transfer integrals that depend not only for a distance between the chains $r_{i, j},\left|H_{i, j}\right| \propto \exp \left(-r_{i, j}\right)$, but also accounts for relative orientations of two chains. (For additional details of ZINDO calculations see the Supporting Information [45].) Note that ZINDO is well known to underestimate the value of the transfer integral. However, for a set of geometries the shape of the distribution of transfer integral looks very similar to the one obtained at higher level of calculation (see, for instance, Ref. [55]). Regarding rotations and translations, many studies have investigated those effects on model systems and show that ZINDO is able to reproduce the trend obtained at higher level on rotations and translations (see, for instance, Ref. [56]). In the case where sites $i$ and $j$ belong to the same chain, we arbitrarily set $\left|H_{i, j}\right|=1 \mathrm{eV}$, which greatly exceed typical values of the intrachain transfer integrals. This is justified because PEDOT chains are relatively short, and hence the MOs are typically delocalized over significant part of the chain as stated previously. As a result, the intramolecular transfer rate in much higher than the intermolecular one, and the limiting step for charge transport through the volume is linked to the efficiency of the intermolecular transport. (a)

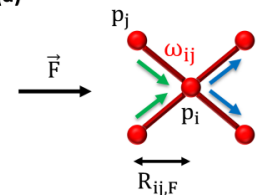

$\mathrm{R}_{\mathrm{ij}, \mathrm{F}}$ : Site distance along the field

F: Electric field value

$\mathrm{p}_{\mathrm{i}, \mathrm{j}} \in[0,1]$ : Occupation probability

$\omega_{i, j}:$ Hopping rate from $i$ to $j$

p: Average occupation probability

$\mathrm{N}_{\mathrm{t}}$ : Number of nodes (b)

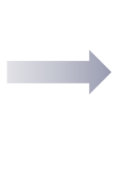

(c)

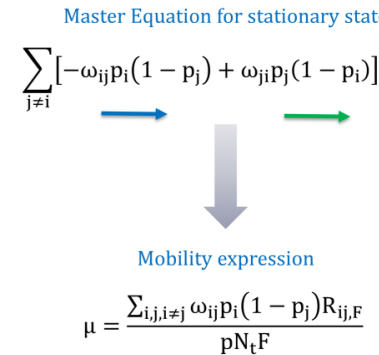

FIG. 2. Workflow to calculate mobility from the resistive network. [(a) and (b)] The stationary state of the carriers under an electric field $F$ is described by the master equation for the occupation probabilities $p_{i}$ at every transport units $i$. Solution of this equation is obtained iteratively; once all $p_{i}$ are calculated, the mobility is accessed via a equation given in (c) [57].

\section{B. From resistive network to mobility}

With these well-defined resistive networks, we finally calculated relative mobilities for the different morphologies, through a mean-field approach as described by Yu et al. [57]. The procedure is depicted in Fig. 2. Each transport unit has an average charge carrier occupation probability $p_{i}$. Under the application of an electric field $F$, chosen to be $10^{4} \mathrm{~V} / \mathrm{cm}$, the charge carriers can hop from a node $i$ to another node $j$ with a given rate $\omega_{i, j}$. The occupation probability $p_{i}$ at each transport unit $i$ in a stationary state $\left(d p_{i} / d t=0\right)$ is described by the so-called master equation, as given in Fig. 2. The solution of this equation is calculated by an iterative process that eventually provides the occupation probabilities for all nodes $i$. With these $p_{i}$ at hand, it is then straightforward to calculate the mobility as shown in Fig. 2 .

As shown in Fig. 2(c), the mean charge carrier occupation $p$, i.e., the number of charge carriers per node, is a parameter that influences the mobility calculation. It is expected that it depends on the oxidation level (33\% in our systems), but it should be stressed that the carrier density and the oxidation level are not the same quantities, and they can differ by orders of magnitude $[20,23,26]$. In our calculations we set the carrier density to $5 \times 10^{20} \mathrm{~cm}^{-3}$ (corresponding to $p=4 \times 10^{-2}$ ), which corresponds to the measurements of the carrier density in PEDOT reported by Park et al. [58]. Note that for high carrier density the mean-field approximation to the master equation misses the true interaction between charge carriers because it only takes into account the Pauli exclusion principle in a mean-field way. However, we do not expect this to affect our results about morphology-mobility dependence presented in this paper, because the charge carrier densities studied here are relatively low $\left(p=4 \times 10^{-2}\right)$. The temperature is set to $T=300 \mathrm{~K}$. Note that mobility calculations were also performed for a range of temperatures around $T=300 \mathrm{~K}$. The results show an activation behavior of the mobility which is typically seen in the experiment [59] and which is expected for the Miller-Abrahams model (see, for instance, Ref. [20]). All these calculations were coded in $\mathrm{C}++$ in an open-source package available on Git [60]. The code inputs are the atom coordinates (corresponding to one specific frame of the time-dependent MD simulation, note that results from 


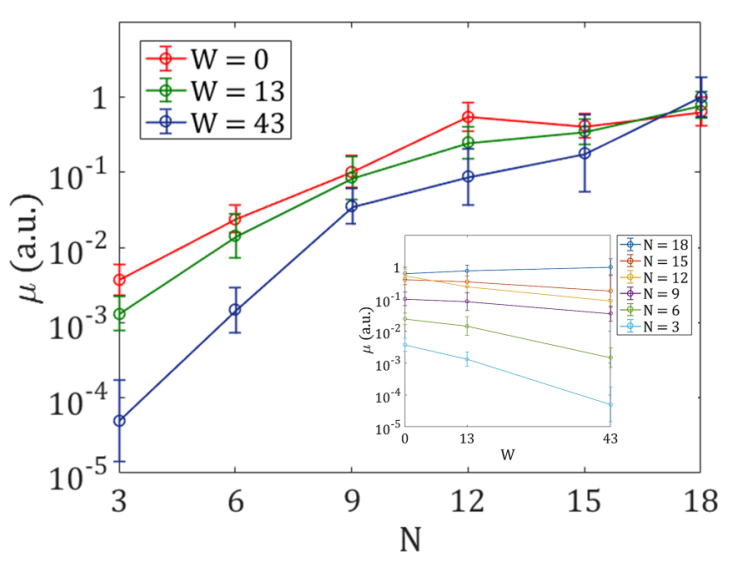

FIG. 3. Dependence of the mobility on the chain length $N$ of the PEDOT oligomers. The inset represents the same data but focusing on the dependence of the mobility on the water content $W$ for different chain length $N$. Points represent values averaged over 13 frames (i.e., different MD simulations) and three electric field directions (along the $x, y$, and $z$ axis) and 100 realizations of the Gaussian DOS, and the error bars are the associated standard deviations.

several frames corresponding to different times of the same MD simulation are gathered and averaged) and the transfer integrals between MOs of the molecules.

The dependence of the mobility on the chain length $N$ and water content $W$ is presented in Fig. 3. The calculated dependence of mobility on the chain length $N$ exhibits the same trend as the experimental curves reported by Noriega et al. [17]: There is a fast increase of the mobility followed by a saturation plateau for the longest chains. However, while in
Ref. [17] the plateau occurs for very long chains with about hundred monomers, in the present study it is already shown for the chain lengths $N \gtrsim 12$. This behavior is present whatever the water content $W$ is. For shorter chains $N \lesssim 12$ the mobility is increased as water is removed. Interestingly, the shorter the chains are, the highest this increase is. However, for the longest chain, $N=18$, the mobility is practically insensitive to the water content and even somehow decreases when $W$ is reduced. To shed some light on this behavior, in the next section we examined the transfer integrals distributions and calculate percolation thresholds for different systems.

\section{Transfer integral and percolation}

A representative transfer integrals distribution is shown in Fig. 4(b), where $P\left(|H|^{2}\right)$ denotes the probability distribution of connections with a given squared transfer integral $|H|^{2}$ scaled by the maximum number of connections with the same transfer integral in the system (i.e., peak C). This distribution exhibits three distinct peaks that can be related to specific morphological features in the system as illustrated in Fig. 4(a). To clarify this relation, we plot [Fig. 4(c)] the transfer integral values against the minimal distance between two polymer chains $d_{\min }$. Additionally, for each chain we introduce a pair of vectors $(\vec{n}, \vec{p})$ which are respectively the normal and parallel vectors to the chain backbone, for the two monomers corresponding to $d_{\min }$, as illustrated in Fig. 4(a). We then introduce an angle $\alpha+\beta$ that characterizes a misalignment between two chains ( $\alpha$ and $\beta$ describe misalignment respectively in the normal direction and in the backbone direction; for definition of $\alpha$ and $\beta$, see Fig. 4). One can see that a low value of $\alpha+\beta$ indicates two chains involved in a $\pi-\pi$ stacking interaction, such as (a)

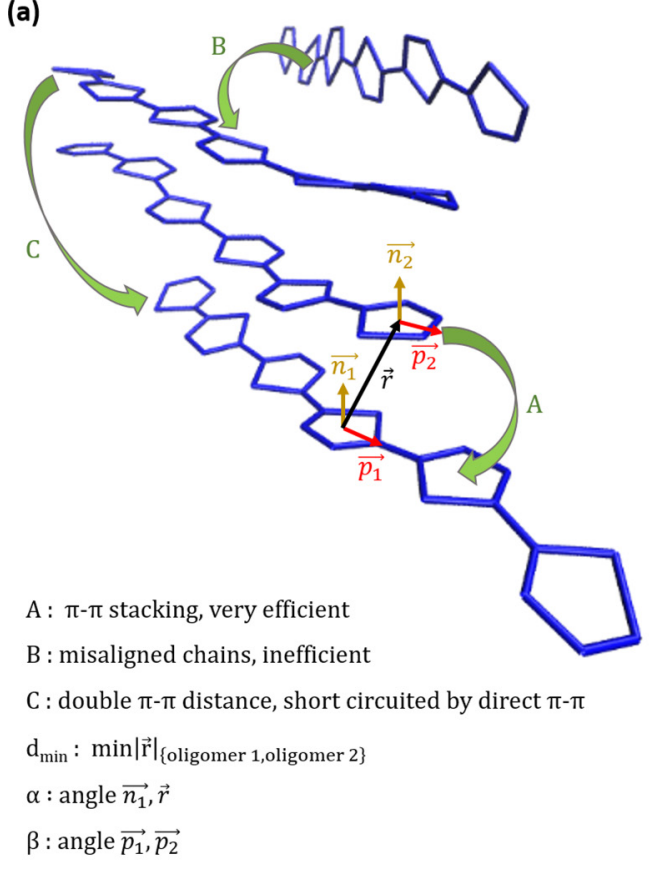

(b)

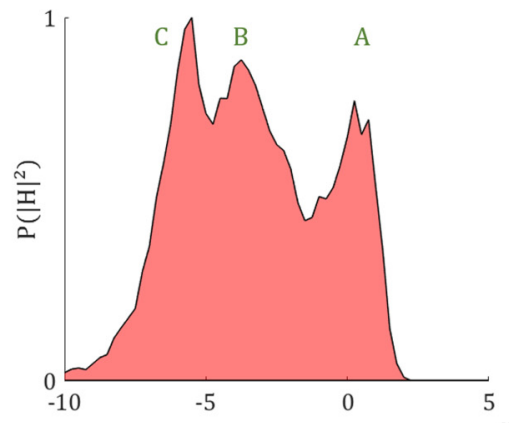

(c)

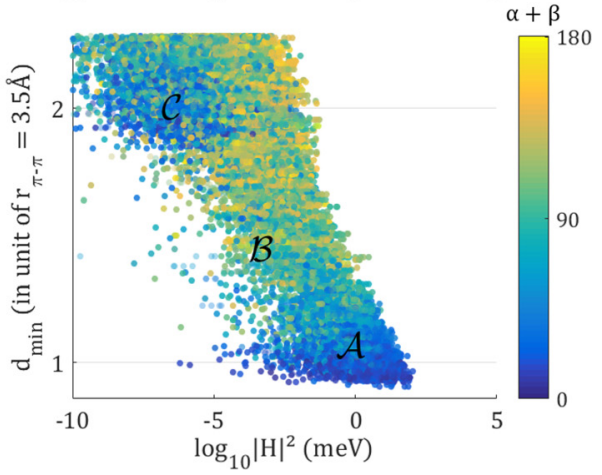

FIG. 4. Correlation between morphology and transfer integrals distribution. (a) Representative molecular dynamics snapshot $(N=6$, $W=0$, only the thiophene rings are represented for clarity) where the three types of possible hops are indicated together with a set of geometrical features. (b) Representative transfer integrals distribution corresponding to the system $N=6$ and $W=0$ (statistics over 13 frames, the distribution is normalized to the maximum value). (c) Correlation between polymers arrangement and transfer integrals values. 
(a)

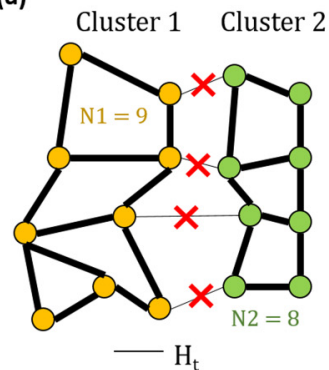

(b)

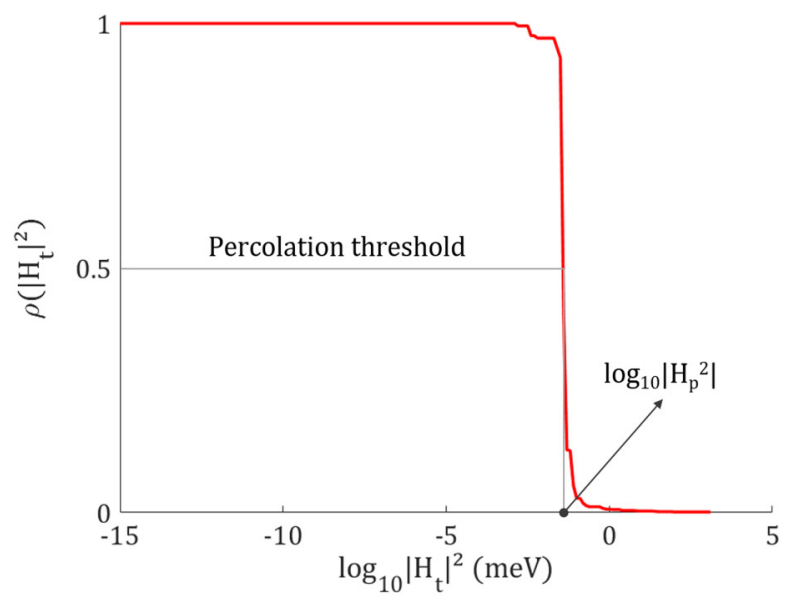

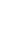

(c)

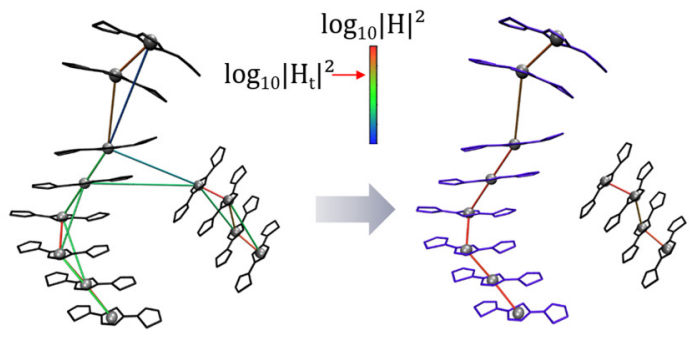

FIG. 5. (a) Calculation of the percolation curve and network clustering. In this example, $\rho=9 /(8+9)=0.53$. (b) Representative percolation curve (chain length $N=3$, water content $W=0$ ). The curve exhibits a steplike behavior defining a so-called percolation threshold that corresponds to an onset for the charge carriers transport through the system. (c) Illustration of the network clustering in a real morphology (chain length $N=3$, water content $W=0$ ). Left: One cluster with color scale indicating the transfer integral values between the nodes. Right: The cluster is divided in two smaller clusters when a threshold is set for the transfer integral.

those connected by A in Fig. 4(a), while a high value denotes misaligned chains such as those connected by B.

Coming back to the plot in Fig. 4(c), we identify there a region (denoted as " $\mathcal{A}$ ") of low values of the angle $\alpha+\beta$ corresponding to the distance between chains of about $r_{\pi-\pi}$, where $r_{\pi-\pi} \approx 3.5 \AA$ is a $\pi-\pi$ stacking distance between chains. Region " $\mathcal{A}$ " corresponds to high transfer integrals values, peak A in Fig. 4(b), which is related to the presence of the efficient $\pi-\pi$ connections in the system. Another region in Fig. 4(c) (denoted as " $\mathcal{C}$ ") of low values of the angle $\alpha+\beta$ is present, but it has much lower transfer integral values (about 5 orders of magnitude). The corresponding distance between chains is around $2 r_{\pi-\pi}$, indicating that this area in the plot is related to the double $\pi-\pi$ stacking in the system. Apparently, peak $\mathrm{C}$ in Fig. 4(b) does not contribute to the transport because such path for the charge carriers is short-circuited by the direct $\pi-\pi$ connection. Last but not least, peak B in Fig. 4(b) appears as a wide distribution of intermediate transfer integrals values, with no particular orientation between chains. As an example in Fig. 4(a), it corresponds to the connection between two distinct $\pi-\pi$ crystallites. Note that a totally amorphous polymer material would exhibit only one peak of this kind (i.e., only peak B).

As it will be shown in the following, the transfer integrals distribution by itself is not sufficient to explain the mobility behavior in our systems. In order to understand how the morphology affects the mobility we combine the analysis of the transfer integrals with a percolation study as introduced by Savoie et al. [61]. In particular, we calculate a so-called percolation curve, see Fig. 5(b) that shows the proportion $\rho$ of chains in the largest cluster as a function of $H_{t}^{2}$. It is calculated by counting a number of chains in the largest cluster when for a given $H_{t}$ all transport edges with the transfer integral below this value, $\left|H_{i j}\right|<H_{t}$, are removed, see Figs. 5(a) and 5(c) for an illustration. The percolation curve exhibits a characteristic steplike behavior providing a specific threshold value $H_{t}^{2}$ of the transfer integral, denoted as $H_{p}^{2}$, for which the proportion of chains in the biggest cluster decreases sharply [see Fig. 5(b)]; the higher this value, the better the mobility. The obtained percolation threshold value $\left|H_{p}\right|^{2}$ of the transfer integral apparently corresponds to an onset for the charge carriers conduction through the entire system (see the Supporting Information for details [45], Fig. S1).

Based on the transfer integrals distributions and percolation curves, one can now fully understand the mobility behaviors depending on the system morphologies. Figure 6(a) shows the transfer integrals distributions for the dry phase for chain lengths $N=3,9$, and 18 . The distributions shift toward lower values of transfer integrals as the chain length increases up to $N \approx 12$ and then stays practically unchanged for $N \gtrsim 15$. This behavior can be clearly seen in Fig. 6(d) which shows an evolution of the peaks A as $N$ changes for the water content $W=0$. (Note that the transfer integrals distributions for the remaining water content of $W=13,43$ as well as a detailed discussion of their behavior is presented in the Supporting Information [45]). Based on the calculated evolution of the distributions one would be tempted to predict a decrease of the mobility with its subsequent saturation $N \gtrsim 15$, which is the opposite of the mobility behavior presented in Fig. 3, where the mobility instead increases and then saturates at $N \gtrsim 12$. This case clearly demonstrates that the transfer integrals distribution by itself is not sufficient to account for the electrical properties of the material. Indeed, one has to compare the percolation curves for the different chain lengths $N$ to understand 

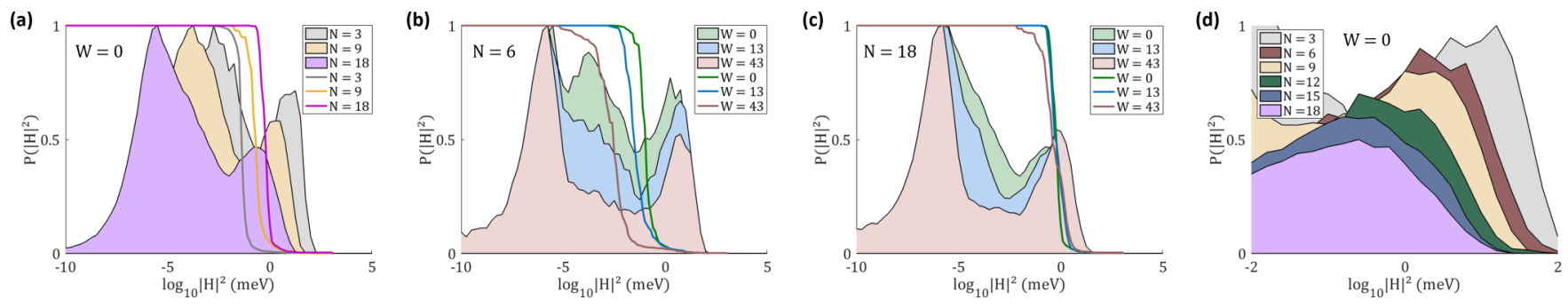

(e)

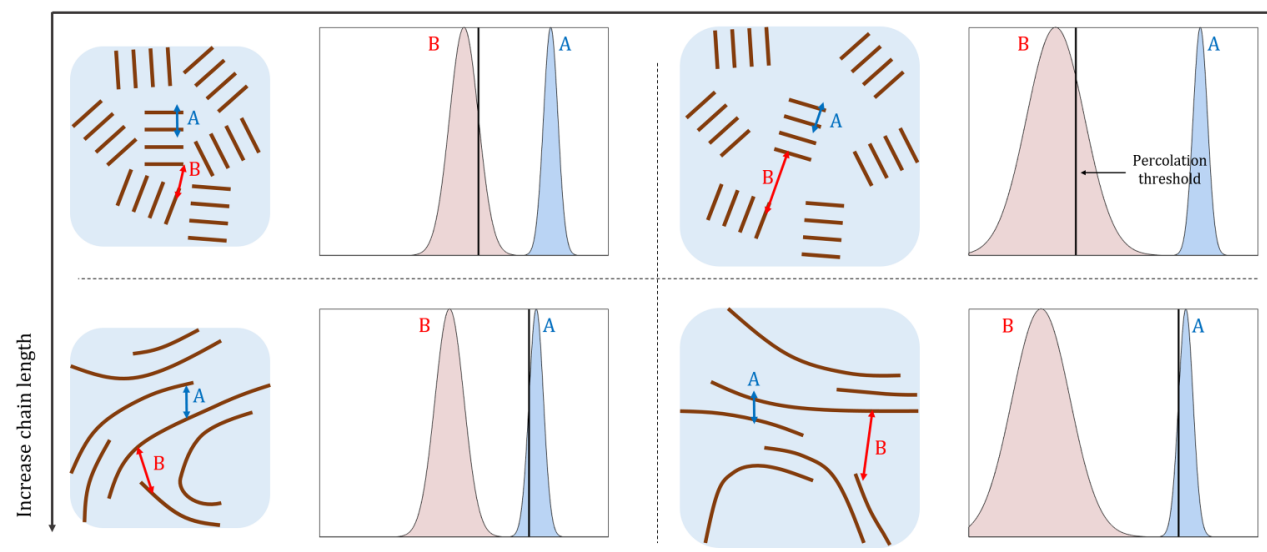

Increase water

FIG. 6. [(a)-(c)] Filled areas correspond to the transfer integrals distributions in various systems (statistics over 13 frames, normalized to the maximum value). Solid lines are the corresponding percolation curves. (d) Evolution of the peak A as $N$ changes for the water content $W=0$. (e) Schematic view of the link between microstructure and transfer integral features.

the increase of the mobility with $N$. The percolation onset is located in the misaligned peak B for the shortest chains $N=3$, and it progressively moves towards the $\pi-\pi$ peak A when $N$ increases, see Fig. 6(a). This behavior is a signature of the system morphology as schematically illustrated in Fig. 6(e). For short chains quasiperfect crystallites are formed with chains stacked over their whole length. Thus the system contains a set of disjoint crystallites and the charge carriers have to hop from one crystallite to another via inefficient connection. As a result, the percolation onset is located close to the misaligned peak $\mathrm{B}$. On the other hand, when the chains become long enough, there are also good $\pi-\pi$ connections but the chains are stacked over a portion of their length only. Thus there is room to connect other chains such that the charge carriers can move throughout the entire system via $\pi-\pi$ connections, and therefore, the percolation onset is located at the $\pi-\pi$ transfer integrals peak A. This results shows that it is the $\pi-\pi$ connection between polymeric chains giving rise to a percolative network through the whole sample, rather than the good crystallinity of the material that ensures good mobility in a polymeric material. This morphological picture is similar to what has been proposed and experimentally confirmed by Kline et al. [62] for poly(3-hexylthiophene) [62].

The mobility plateau occurring for $N \gtrsim 12$ is also well understood within this percolation study. As we demonstrated above, when sufficient $\pi-\pi$ connectivity is reached in the system (i.e., for the long enough chains), the percolation onset is located at the $\pi-\pi$ peak $\mathrm{A}$ and and stays there as $N$ increases. This behavior, combined with the fact that the position of the A peak does not change when the chain length increases above $N \approx 12$ leads to the saturation of the mobility in the system.
The mobility dependence with the water content can be understood considering the evolution of the misaligned and $\pi-\pi$ transfer integrals peaks (B and A), as shown in Figs. 6(b) and 6(c). Regardless the chain length, we observe that peak A is unaffected by the water content: $\pi-\pi$ stacking interaction is an intrinsic property of the polymer and does not depend on the water content because the distance between two chains and their orientation in this configuration is the same. On the other hand, peak B corresponds to misaligned chains from different $\pi-\pi$ crystallites, for which the distance strongly depends on the water content, as for instance depicted in Fig. 6(e) where small crystallites get diluted on water addition. As a consequence, this peak shifts towards lower values of the transfer integral and broadens when $W$ increases. Now for the short chains $N=3$, since the percolation onset "sticks" to the misaligned peak B, it also shifts towards lower values of the transfer integral and the mobility will decrease when $W$ increases. In contrast, for the chains $N=18$, the percolation onset "sticks" to the $\pi-\pi$ stacking peak that remains unaffected by the water content; as a result the mobility remains constant. Therefore, monitoring the mobility as a function of a polymer material hydration may be an experimental way to assess the quality of the connectivity in the system.

\section{CONCLUSION}

A model was developed to investigate the link between morphology and mobility in PEDOT:Tos. This model involved molecular dynamics simulations to generate realistic polymer morphologies, transformation of these morphologies into resistive networks, and calculation of relative mobilities through 
a mean-field approach. Different chain lengths ranging from $N=3$ monomers per chain to $N=18$ monomers per chain were investigated, as well as water content $W=0,13$ and $43 \%$ w/w. An exponential enhancement of the mobility with the chain length has been shown, until a plateau is reached for the longest chains $N \gtrsim 15$. This behavior has been explained in terms of the transfer integrals distributions and percolation thresholds and confirms the experimental evidence showing that, on increasing chain length, the morphology evolves from a set of well-defined but disconnected crystallites to a lessordered material that exhibits an efficient network of $\pi-\pi$ stacking extending throughout the material. Interestingly, the water content affects the mobility depending on the chain length. For the shortest chains, without good percolative networks, increasing the water content drastically reduces the mobility while the effect of water becomes negligible when sufficient connection through $\pi-\pi$ stacking is reached for longer chains $N \gtrsim 15$. Our study provides an answer to the long-debated question on the importance of crystallinity and the long-range order for enhancement of the performance of polymeric materials. Namely, we demonstrate that it is the $\pi-\pi$ connections between polymeric chains giving rise to a percolative network through the whole sample rather than the good crystallinity of the material that ensure good mobility in conducting polymers. Finally, this study provides a general framework to discuss polymer morphology engineering in term of connectivity, transfer integral, and percolation onset, and the generic model presented opens the door to further investigations, including complex chain length distributions, other dopant and solvent species, and other polymer systems.

\section{ACKNOWLEDGMENTS}

This work was supported by the Swedish Energy Agency (Grants No. 38332-1 and No. 43561-1) and the Knut and Alice Wallenberg Foundation through the project The Tail of the Sun. I.V.Z. thanks the Advanced Functional Material center at Linköping University. We acknowledge the support from the Swedish Research Council via "Research Environment grant" (Grant No. 2016-05990). The computations were performed on resources provided by the Swedish National Infrastructure for Computing (SNIC) at NSC and HPC2N. M.L. thanks SeRC (Swedish e-Science Research Center) for funding.
[1] S. R. Forrest, Nature 428, 911 (2004).

[2] Z. He, C. Zhong, S. Su, M. Xu, H. Wu, and Y. Cao, Nat. Photon. 6, 591 (2012).

[3] H. Sirringhaus, Adv. Mater. 26, 1319 (2014).

[4] J. Rivnay, P. Leleux, M. Ferro, M. Sessolo, A. Williamson, D. A. Koutsouras, D. Khodagholy, M. Ramuz, X. Strakosas, R. M. Owens, C. Benar, J.-M. Badier, C. Bernard, and G. G. Malliaras, Sci. Adv. 1, e1400251 (2015)

[5] O. Inganas, Nat. Photon. 5, 201 (2011).

[6] Z.-T. Zhu, J. T. Mabeck, C. Zhu, N. C. Cady, C. A. Batt, and G. G. Malliaras, Chem. Commun. 13, 1556 (2004).

[7] O. Bubnova, Z. U. Khan, A. Malti, S. Braun, M. Fahlman, and M. X. Berggren, Nat. Mater. 10, 429 (2011).

[8] H. Shi, C. Liu, Q. Jiang, and J. Xu, Adv. Electr. Mater. 1, 1500017 (2015).

[9] N. Kim, H. Kang, J.-H. Lee, S. Kee, S. H. Lee, and K. Lee, Adv. Mater. 27, 2317 (2015).

[10] O. Bubnova et al. ,Nat. Mater. 13, 190 (2014).

[11] N. Kim, B. H. Lee, D. Choi, G. Kim, H. Kim, J.-R. Kim, J. Lee, Y. H. Kahng, and K. Lee, Phys. Rev. Lett. 109, 106405 (2012).

[12] M. V. Fabretto, D. R. Evans, M. Mueller, K. Zuber, Hojati-P. Talemi, R. D. Short, G. G. Wallace, and P. J. Murphy, Chem. Mater. 24, 3998 (2012).

[13] A. Williamson, J. Rivnay, L. Kergoat, A. Jonsson, S. Inal, I. Uguz, M. Ferro, A. Ivanov, T. A. Sjöström, D. T. Simon, M. Berggren, G. G. Malliaras, and C. Bernard, Adv. Mater. 27, 3138 (2015).

[14] A. Jonsson, Z. Song, D. Nilsson, B. A. Meyerson, D. T. Simon, B. Linderoth, and M. Berggren, Sci. Adv. 1, e1500039 (2015).

[15] A. Malti, J. Edberg, H. Granberg, Z. U. Khan, J. W. Andreasen, X. Liu, D. Zhao, H. Zhang, Y. Yao, J. W. Brill, I. Engquist, M. Fahlman, L. Wågberg, X. Crispin, and M. Berggren, Adv. Sci. 3, 1500305 (2016).
[16] A. M. Bryan, L. M. Santino, Y. Lu, S. Acharya, and J. M. D’ Arcy, Chem. Mater. 28, 5989 (2016).

[17] R. Noriega, J. Rivnay, K. Vandewal, F. P. V. Koch, N. Stingelin, P. Smith, M. F. Toney, and A. Salleo, Nat. Mater. 12, 1038 (2013).

[18] X. Zhang, H. Bronstein, A. J. Kronemeijer, J. Smith, Y. Kim, R. J. Kline, L. J. Richter, T. D. Anthopoulos, H. Sirringhaus, K. Song, M. Heeney, W. Zhang, I. McCulloch, and D. M. DeLongchamp, Nat. Commun. 4, 2238 (2013)..

[19] S. Wang, S. Fabiano, S. Himmelberger, S. Puzinas, X. Crispin, A. Salleo, and M. Berggren, Proc. Natl. Acad. Sci. U.S.A. 112, 10599 (2015).

[20] S. Ihnatsenka, X. Crispin, and I. V. Zozoulenko, Phys. Rev. B 92, 035201 (2015).

[21] D. Mendels and N. Tessler, J. Phys. Chem. C 117, 3287 (2013).

[22] R. Schmechel, J. Appl. Phys. 93, 4653 (2003).

[23] G. Kim and K. P. Pipe, Phys. Rev. B 86, 085208 (2012).

[24] E.-G. Kim and J.-L. Brédas, J. Am. Chem. Soc. 130, 16880 (2008).

[25] B. Zhang, K. Wang, D. Li, and X. Cui, RSC Adv. 5, 33885 (2015).

[26] W. Shi, T. Zhao, J. Xi, D. Wang, and Z. Shuai, J. Am. Chem. Soc. 137, 12929 (2015).

[27] S. D. Kang and G. J. Snyder, Nat. Mater. 16, 252 (2017).

[28] J. Kirkpatrick, V. Marcon, J. Nelson, K. Kremer, and D. Andrienko, Phys. Rev. Lett. 98, 227402 (2007).

[29] A. Troisi, Adv. Mater. 19, 2000 (2007).

[30] J. Nelson, J. J. Kwiatkowski, J. Kirkpatrick, and J. M. Frost, Acc. Chem. Res. 42, 1768 (2009).

[31] V. Rühle, J. Kirkpatrick, and D. Andrienko, J. Chem. Phys. 132, 134103 (2010).

[32] T. Vehoff, B. Baumeier, A. Troisi, and D. Andrienko, J. Am. Chem. Soc. 132, 11702 (2010) 
[33] L. Wang, Q. Li, Z. Shuai, L. Chen, and Q. Shi, Phys. Chem. Chem. Phys. 12, 3309 (2010).

[34] L. Wang, G. Nan, X. Yang, Q. Peng, Q. Li, and Z. Shuai, Chem. Soc. Rev. 39, 423 (2010).

[35] V. Rühle, A. Lukyanov, F. May, M. Schrader, T. Vehoff, J. Kirkpatrick, B. Baumeier, and D. Andrienko, J. Chem. Theory Comput. 7, 3335 (2011).

[36] M. Jakobsson, M. Linares, and S. Stafström, J. Chem. Phys. 137, 114901 (2012).

[37] Z. Shuai, H. Geng, W. Xu, Y. Liao, and J.-M. André, Chem. Soc. Rev. 43, 2662 (2014).

[38] R. Volpi, S. Stafström, and M. Linares, J. Chem. Phys. 142, 094503 (2015).

[39] R. Volpi, S. Kottravel, M. S. Nørby, S. Stafström, and M. Linares, J. Chem. Theory Comput. 12, 812 (2016).

[40] I. Yavuz, B. N. Martin, J. Park, and K. N. Houk, J. Am. Chem. Soc. 137, 2856 (2015).

[41] X. Feng, V. Marcon, W. Pisula, M. R. Hansen, J. Kirkpatrick, F. Grozema, D. Andrienko, K. Kremer, and K. Müllen, Nat. Mater. 8, 421 (2009).

[42] J. F. Franco-Gonzalez and I. V. Zozoulenko, J. Phys. Chem. B 121, 4299 (2017).

[43] J. Felipe Franco-Gonzalez, E. Pavlopoulou, E. Stavrinidou, R. Gabrielsson, T. D. Simon, M. Berggren, and I. V. Zozoulenko, Nanoscale 9, 13717 (2017).

[44] S. Rudd, J. F. Franco-Gonzalez, K. S. Singh, Z. U. Khan, X. Crispin, J. W. Andreasen, I. Zozoulenko, and D. Evans, J. Polym. Sci. Part B: Polym. Phys. 56, 97 (2017).

[45] See Supplemental Material at http://link.aps.org/supplemental/ 10.1103/PhysRevMaterials.2.045605 for computational details to obtain the morphologies generated with molecular dynamics simulations and computational details to solve the master equation for transport and to generate the percolation curves. Figure S1 illustrates the percolation concept and gives details about the transfer integral calculation. Figure S2 compares transfer integral distributions obtained with different levels of theory. Figure S3 shows the saturation of the - shift when N increases. We also provide a movie showing a morphology and the resistive network for a system with $\mathrm{N}=6$ and $\mathrm{W}=13$.

[46] I. Petsagkourakis, E. Pavlopoulou, G. Portale, B. A. Kuropatwa, S. Dilhaire, G. Fleury, and G. Hadziioannou, Sci. Rep. 6, 30501 (2016).

[47] A. Elschner, PEDOT: Principles and Applications of an Intrinsically Conductive Polymer (CRC Press, Boca Raton, FL, 2011)

[48] A. Ugur, F. Katmis, M. Li, L. Wu, Y. Zhu, K. K. Varanasi, and K. K. Gleason, Adv. Mater. 27, 4604 (2015).

[49] W. Humphrey, A. Dalke, and K. Schulten, J. Molec. Graph. 14, 33 (1996).

[50] W. A. Muñoz, S. K. Singh, J. F. Franco-Gonzalez, M. Linares, X. Crispin, and I. V. Zozoulenko, Phys. Rev. B 94, 205202 (2016).

[51] W. A. Muñoz, X. Crispin, M. Fahlman, and I. V. Zozoulenko, Macromolec. Rapid Commun. 39, 1700533 (2017).

[52] A. Miller and E. Abrahams, Phys. Rev. 120, 745 (1960).

[53] J. Kirkpatrick, Int. J. Quantum Chem. 108, 51 (2008)

[54] M. J. Frisch et al., Gaussian09 Revision E.01 (Gaussian Inc., Wallingford CT, 2009).

[55] L. Sousa, R. Volpi, D. A. da Silva Filho, and M. Linares, Chem. Phys. Lett. 689, 74 (2017).

[56] R. C. I. MacKenzie, J. M. Frost, and J. Nelson, J. Chem. Phys. 132, 064904 (2010).

[57] Z. G. Yu, D. L. Smith, A. Saxena, R. L. Martin, and A. R. Bishop, Phys. Rev. B 63, 085202 (2001).

[58] H.-S. Park, S.-J. Ko, J.-S. Park, J. Y. Kim, and H.-K. Song, Sci. Rep. 3, 2454 (2013).

[59] O. Bubnova, M. Berggren, and X. Crispin, J. Am. Chem. Soc. 134, 16456 (2012).

[60] https://github.com/nicro31/LOE-CTP.

[61] B. M. Savoie, K. L. Kohlstedt, N. E. Jackson, L. X. Chen, M. O. d. 1. Cruz, G. C. Schatz, T. J. Marks, and M. A. Ratner, Proc. Natl. Acad. Sci. U.S.A. 111, 10055 (2014).

[62] R. J. Kline, M. D. McGehee, E. N. Kadnikova, J. Liu, J. M. J. Fréchet, and M. F. Toney, Macromolecules 38, 3312 (2005). 